\title{
Contents
}

1 Quantum Phase Transitions of Antiferromagnets

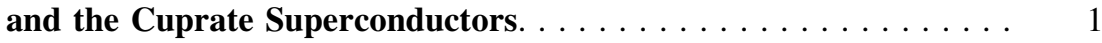

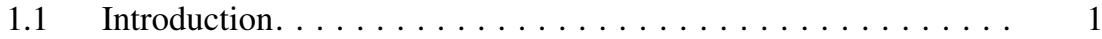

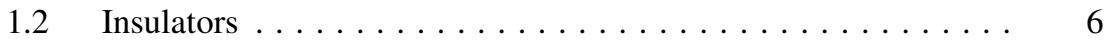

1.2.1 Coupled Dimer Antiferromagnets: Bond Operators . . . 6

1.2.2 Frustrated Square Lattice Antiferromagnets:

Schwinger Bosons . . . . . . . . . . . . . . . . . 13

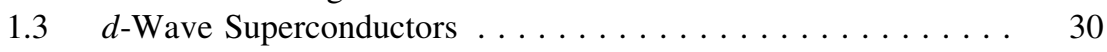

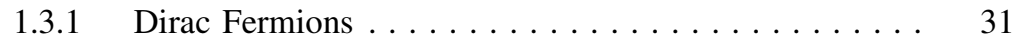

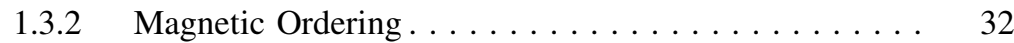

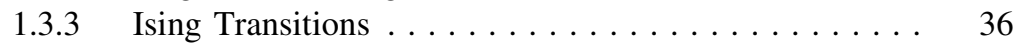

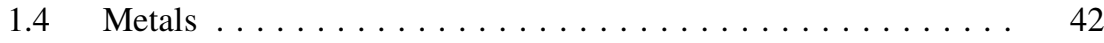

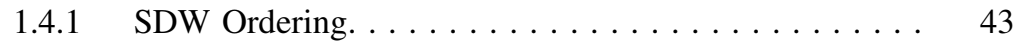

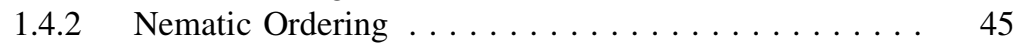

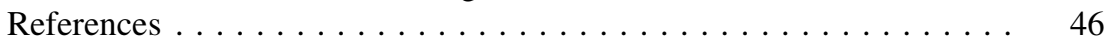

\section{Electronic Liquid Crystal Phases in Strongly}

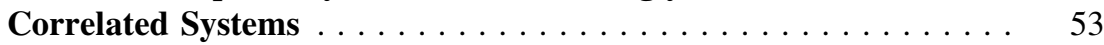

$2.1 \quad$ Electronic Liquid Crystal Phases . . . . . . . . . . . . . 53

2.1.1 Symmetries of Electronic Liquid Crystal Phases . . . . . 54

2.1.2 Order Parameters and Their Symmetries. . . . . . . . 56

2.1.3 Electronic Liquid Crystal Phases and Strong Correlation Physics . . . . . . . . . . . . . . 58

2.2 Experimental Evidence in Strongly Correlated Systems . . . . . 60

2.2.1 Nematic Phases in the 2DEG in High Magnetic Fields . . . . . . . . . . . . . . . . 60

2.2.2 The Nematic Phase of $\mathrm{Sr}_{3} \mathrm{Ru}_{2} \mathrm{O}_{7} \ldots \ldots \ldots 62$

2.2.3 Stripe Phases and Nematic Phases in the Cuprates.... 63

2.2.4 Conventional CDW Materials ............. 70 
2.3 Theories of Stripe Phases . . . . . . . . . . . . 71

2.3.1 Stripe Phases in Microscopic Models . . . . . . . . 71

2.3.2 Phases of Stripe States . . . . . . . . . . . . . . 74

2.4 Is Inhomogeneity Good or Bad for Superconductivity?. . . . . . 79

2.5 The Striped Superconductor: A Pair Density Wave State .... . 81

2.5.1 Landau-Ginzburg Theory of the Pair Density Wave. . 83

2.5.2 Charge 4e SC Order and the Topological Excitations of the PDW SC State . . . . . . . . . . . . . 85

2.6 Nematic Phases in Fermi Systems . . . . . . . . . . . . . 87

2.6.1 The Pomeranchuk Instability . . . . . . . . . . . . . 87

2.6.2 The Nematic Fermi Fluid . . . . . . . . . . . . . . . . 89

2.7 Generalizations: Unconventional Magnetism

and Time Reversal Symmetry Breaking . . . . . . . . . . . 95

2.8 Nematic Order in the Strong Correlation Regime. . . . . . . . . 98

2.9 The Quantum Nematic-Smectic QCP and the Melting

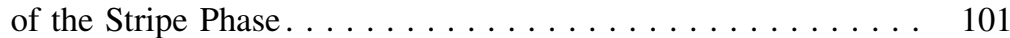

2.10 Outlook ............................ 105

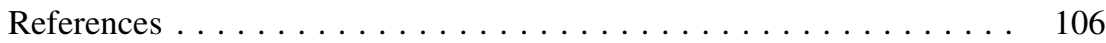

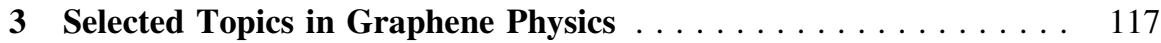

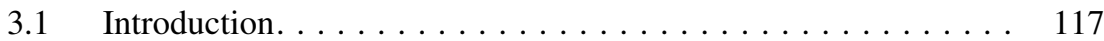

3.2 The Chemistry. . . . . . . . . . . . . . . . . . . . 119

3.3 The Crystal and Band Structure. . . . . . . . . . . . . . 123

3.4 Phonons in Free Floating Graphene . . . . . . . . . . . . . . 129

3.5 Constrained Graphene. . . . . . . . . . . . . . . . . 132

3.6 Deformed Graphene. . . . . . . . . . . . . . . . . 135

3.6.1 A Non-Trivial Example: The Scroll . . . . . . . . . . . 139

3.7 Conclusions. . . . . . . . . . . . . . . . . . . . . 142

References ............................. 143

4 Strong Electronic Correlations: Dynamical Mean-Field Theory

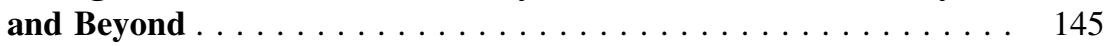

4.1 The Physics of Strongly Correlated Materials . . . . . . . . . 146

4.1.1 Theoretical Description. . . . . . . . . . . . . . . . 147

4.2 The Dynamical Mean-Field Theory Construction:

An Embedded Atom. . . . . . . . . . . . . . . . . . . . . 148

4.2.1 A Reminder on Classical Mean-Field Theory . . . . . . 149

4.2.2 Generalization to the Quantum Case: Dynamical Mean-Field Theory. . . . . . . . . . . . . . 150

4.2.3 The Embedded Atom as an Anderson Impurity Model . . . . . . . . . . . . . . . . . . . . . 152

4.2.4 The Self-Consistency Loop in Practice......... . 152 
4.3 The Metal to Mott Insulator Transition from a DMFT Perspective. . . . . . . . . . . . . . . . . . 153

4.4 From Models to Real Materials: Combining DMFT with First-Principle Electronic Structure Methods . . . . . . . . 156

$4.4 .1 \quad$ Illustrations . . . . . . . . . . . . . . . . . . . 162

4.5 Efficient Algorithms for Solving Multiorbital

Quantum Impurity Problems . . . . . . . . . . . . . . . . . . . . . . 164

4.5.1 Interaction Expansion Algorithm . . . . . . . . . . . . . 164

4.5.2 Monte Carlo Sampling . . . . . . . . . . . . . . . . . 167

4.5.3 Fast Update Formulae. . . . . . . . . . . . . . . . . . . . 167

4.5.4 Measurement of Green's Function . . . . . . . . . . . . . . . . . . . . . . 169

4.5.5 Sign Problem and $\alpha$-Parameters . . . . . . . . . . . . . 172

4.5.6 Hybridization Expansion Algorithm . . . . . . . . . . . . 172

4.5.7 Measurement of Green's Function . . . . . . . . . . . . . . 176

4.6 Cluster Extensions of DMFT: Short-Range

Non-Local Correlations. . . . . . . . . . . . . . . . . . . . . . . . . . 177

4.7 Long-Range Correlations and the Dual-Fermion

Perturbation Expansion . . . . . . . . . . . . . . . . . 182

$4.7 .1 \quad$ Formalism. . . . . . . . . . . . . . . . . . . . . 184

4.7.2 Dual Perturbation Theory . . . . . . . . . . . . . . 187

4.7 .3 Exact Relations . . . . . . . . . . . . . . . . . . . . . . . . 188

4.7.4 Self-Consistency Condition and Relation to DMFT . . . 190

4.7.5 Numerical Results . . . . . . . . . . . . . . . . . . . . 192

4.7.6 Generalizations of the Dual Fermion Approach . . . . . 195

4.7.7 Cluster Dual Fermion Approach . . . . . . . . . . . . . 195

4.7 .8 Calculation of Susceptibilities . . . . . . . . . . . 201

4.7.9 Ladder Approximation . . . . . . . . . . . . . . . . . . . . . . . 204

4.7.10 Convergence Properties ... . . . . . . . . . . . 207

References ........................... 209

\section{Nonequilibrium Transport and Dephasing}

in Coulomb-Blockaded Quantum Dots . . . . . . . . . . . . . . 215

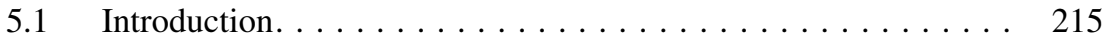

5.2 Coulomb Blockade Phenomena in Quantum Dots . . . . . . . . 216

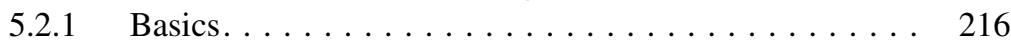

$5.2 .2 \quad$ Energy Scales . . . . . . . . . . . . . . . . 218

5.2.3 Coulomb Blockade and Transport Regimes. . . . . . . . 219

5.2.4 Kondo Effect in Quantum Dots . . . . . . . . . . . . . 221

$5.3 \quad$ Keldysh Approach . . . . . . . . . . . . . . . . . . . . . . . . . . . . 224

$5.3 .1 \quad$ Basic Idea. . . . . . . . . . . . . . . . . . . . . . 224

5.3 .2 Boson Mode . . . . . . . . . . . . . . . . . . 226

5.3.3 Fermion Mode. . . . . . . . . . . . . . . . 230 
5.4 Ambegaokar-Eckern-Schön Action

Within Keldysh Approach. . . . . . . . . . . . . . 232

$5.4 .1 \quad$ Model. . . . . . . . . . . . . . . . . . . . . 232

$5.5 \quad$ Nonequilibrium Dephasing . . . . . . . . . . . . . . . 237

5.5 .1 Tunneling Density of States . . . . . . . . . . . 237

5.5.2 Strong Tunneling: Open Dot . . . . . . . . . . . . . . 239

5.6 Conclusions. . . . . . . . . . . . . . . . . . . . . . . 242

References ............................. 243

6 Many-Body Physics from a Quantum Information Perspective . . 245

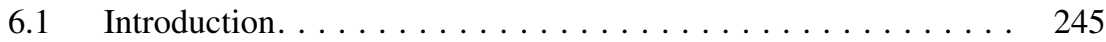

6.2 Aspects of Quantum Information . . . . . . . . . . . . . . 246

6.2.1 Bipartite Pure States: Schmidt Decomposition. . . . . . 247

6.2.2 Bipartite Mixed States: Separable and Entangled States . . . . . . . . . . . . . . . . . . . . 248

6.2.3 Entanglement Criteria. . . . . . . . . . . . . . . . . 249

6.2 .4 Partial Transposition. . . . . . . . . . . . . . . . 249

6.2.5 Entanglement Witnesses from the Hahn-Banach Theorem. . . . . . . . . . . . 251

6.2.6 Positive Maps and the Entanglement Problem . . . . . . 253

6.2.7 Positive Maps and Entanglement Witnesses:

Further Characterization and Examples. . . . . . . 256

6.2 .8 Entanglement Measures . . . . . . . . . . . . . 258

6.3 Area Laws . . . . . . . . . . . . . . . . . . . . . 261

6.3.1 Mean Entanglement of Bipartite States. . . . . . . . . 261

6.3.2 Area Laws in a Nutshell . . . . . . . . . . . . . . . . . . . . 264

6.4 The Tensor Network Product World. . . . . . . . . . . . . . . . . 272

6.4.1 The Tensor Network Representation of Quantum States . . . . . . . . . . . . . . 273

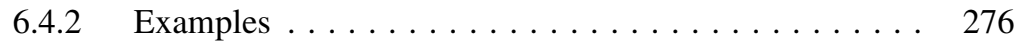

6.4.3 Classical Kinetic Models. . . . . . . . . . . . . . . . . . 279

6.5 Quantum Kinetic Ising Models . . . . . . . . . . . . . . . . . 281

$6.5 .1 \quad$ A Two Spin Flip Model . . . . . . . . . . . . . . . 281

6.5.2 The Single Flip Model . . . . . . . . . . . . . . . . 285

6.6 Discussion and Outlook . . . . . . . . . . . . . . . 287

References ............................. 289

7 Statistical Mechanics of Classical and Quantum

Computational Complexity . . . . . . . . . . . . . . . . 295

$7.1 \quad$ Introduction. . . . . . . . . . . . . . . . . . . . . . . 296

$7.2 \quad$ Complexity Theory for Physicists . . . . . . . . . . . . . . . . . . . 298

7.2.1 Problems, Instances, Computers and Algorithms . . . . 299

7.2.2 Polynomial Reductions and Worst-Case Behavior . . . . 301

7.2.3 Classical: P and NP . . . . . . . . . . . . . . 302 
7.2.4 Quantum: BQP and QMA............... 303

7.2.5 NP-Completeness: Cook-Levin . . . . . . . . . . . 305

7.2.6 QMA-Completeness: Kitaev . . . . . . . . . . . . . . . 307

7.3 Physics for Complexity Theory . . . . . . . . . . . . . . . . . . . 312

7.3.1 Typical Versus Worst-Case Complexity . . . . . . . . . 313

7.3.2 Classical Statistical Mechanics of $\boldsymbol{k}$-SAT . . . . . . . 313

7.3.3 Schematic Phase Diagram of Classical Random $k$-SAT . . . . . . . . . . . . . . . 315

7.3 .4 Cavity Analysis . . . . . . . . . . . . . . . 317

7.3.5 Adiabatic Quantum Algorithm for Classical $k$-SAT . . 320

7.4 Statistical Mechanics of Random $k$-QSAT . . . . . . . . . . . . . . . . . 322

7.4.1 Random $k$-QSAT Ensemble. . . . . . . . . . . . . 322

7.4 .2 Phase Diagram . . . . . . . . . . . . . . . . . . . 323

7.4.3 Geometrization Theorem. . . . . . . . . . . . . . . . . 324

7.4.4 A Few Details of Phases and Transitions . . . . . . . . 325

7.4.5 Satisfying the Promise . . . . . . . . . . . . . . 329

7.4.6 Open Questions . . . . . . . . . . . . . . . . . 330

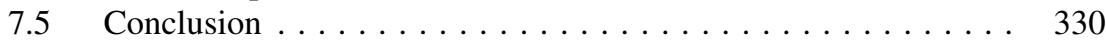

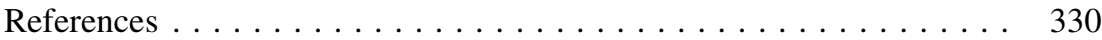

8 Non-Perturbative Methods in (1+1) Dimensional Quantum

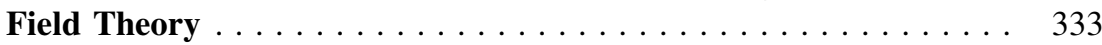

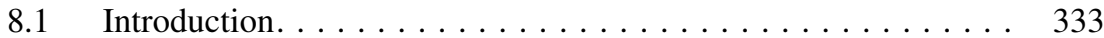

8.2 Functional Form of the Free Energy. . . . . . . . . . . . . . . . 334

8.2.1 Critical Exponents and Universal Ratios . . . . . . . . . 336

8.3 General Properties of the Form Factors . . . . . . . . . . . . . . . . . 338

8.3.1 Faddeev-Zamolodchikov Algebra. . . . . . . . . . . . 339

8.3 .2 Form Factors . . . . . . . . . . . . . . . . . . . . 340

8.4 Watson's Equations . . . . . . . . . . . . . . . . . . . 341

8.5 Recursive Equations . . . . . . . . . . . . . . . . . . . . . . . . . . . 344

8.6 The Operator Space . . . . . . . . . . . . . . . . . . . . . 346

8.7 Correlation Functions . . . . . . . . . . . . . . . . . . . . . . . . 347

8.8 Form Factors of the Stress-Energy Tensor . . . . . . . . . . . 350

8.9 Ultraviolet Limit . . . . . . . . . . . . . . . . . . . . . . . . . . 352

8.10 The Ising Model at $T \neq T_{c} \ldots \ldots \ldots \ldots \ldots \ldots \ldots$

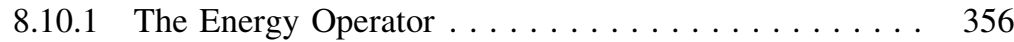

8.10 .2 Magnetization Operators . . . . . . . . . . . . 357

8.10 .3 The Painlevé Equation . . . . . . . . . . . . . . . . 360

8.11 The Ising Model in a Magnetic Field . . . . . . . . . . . . . . . . . . . . . . . . . . . . . . 362

8.12 Conclusions. . . . . . . . . . . . . . . . . . . . . . 367

References ............................. 367 